\title{
Bienestar psicológico y desempeño docente en la educación básica regular
}

\author{
Psychological well-being and teacher performance in regular basic education \\ Bem-estar psicológico e atuação docente no ensino fundamental regular
}

Rachel Vargas Navarro

ravana0564@gmail.com

https://orcid.org/0000-0002-7522-4944

Universidad César Vallejo, Lima-Perú

Gloria Idrogo Barboza

gloriaidrogobarboza29@gmail.com

https://orcid.org/0000-0001-7881-7289

Universidad Nacional de Educación Enrique Guzmán

\author{
Yesica Mabel Aguirre Idrogo \\ yesica_mabela@hotmail.com \\ https://orcid.org/0000-0001-6125-7481 \\ Universidad César Vallejo, Lima-Perú
}

Gina Paola Noel Bravo

ginin.79@gmail.com

https://orcid.org/0000-0001-6334-9785

Universidad César Vallejo, Lima-Perú

y Valle, Lima-Perú

\section{RESUMEN}

La presente investigación tuvo como objetivo, establecer la relación entre el bienestar psicológico y el desempeño de los docentes, en una institución educativa del cono Sur de Lima, 2019. Para esto, se utilizó una metodología con enfoque cuantitativo, de tipo básico. El diseño de investigación fue no experimental, correlacional y de corte transversal. La población estuvo conformada por 90 docentes y la muestra fue censal. Se utilizó la técnica de la encuesta y como instrumentos, fueron utilizados dos cuestionarios, los cuales fueron validados por juicio de expertos y demostrado su confiabilidad, mediante una prueba piloto realizada a 30 docentes. Según el análisis de resultados, se concluye que con respecto a la variable bienestar psicológico y desempeño docente, que existe una correlación positiva $y$ moderada entre las variables, ya que se obtuvo el Rho ,575, y la significancia de p-valor $=, 000$ que es menor a $\alpha=, 05$ por lo cual se rechaza la hipótesis nula.

Palabras clave: Capacidades pedagógicas; Emocionalidad; Relaciones interpersonales
ABSTRACT

The objective of this research was to establish the relationship between psychological well-being and the performance of teachers, in an educational institution in the Southern Cone of Lima, 2019. For this, a methodology with a quantitative approach, of a basic type, was used. The research design was non-experimental, correlational, and cross-sectional. The population consisted of 90 teachers and the sample was census. The survey technique was used and as instruments, two questionnaires were used, which were validated by expert judgment and their reliability demonstrated through a pilot test carried out on 30 teachers. According to the analysis of results, it is concluded that with respect to the psychological well-being and teacher performance variable, that there is a positive and moderate correlation between the variables, since the Rho, 575 , and the significance of $\mathrm{p}$-value $=$ .000 were obtained which is less than $a$ $=.05$, which is why the null hypothesis is rejected.

Key words: Pedagogical skills; Emotionality; Interpersonal relationships

\section{RESUMO}

O objetivo desta pesquisa foi estabelecer a relação entre o bem-estar psicológico e o desempenho de professores, em uma instituição de ensino do Cone Sul de Lima, 2019. Para isso, foi utilizada uma metodologia com abordagem quantitativa, de tipo básico. Usado. $\mathrm{O}$ desenho da pesquisa foi não experimental, correlacional e transversal. A população foi constituída por 90 professores e a amostra foi censitária. Foi utilizada a técnica de survey e como instrumentos foram utilizados dois questionários, os quais foram validados por julgamento de especialistas e sua confiabilidade demonstrada por meio de um teste piloto realizado em 30 professores. De acordo com a análise dos resultados, conclui-se que no que diz respeito à variável bem-estar psicológico e desempenho docente, existe uma correlação positiva e moderada entre as variáveis, uma vez que o Rho, 575, e a significância do $\mathrm{p}$-valor $=.000$ foram obtidos, o que é menor que $\alpha=.05$, razão pela qual a hipótese nula é rejeitada.

Palavras-chave:

Competências pedagógicas; Emocionalidade; Relacionamento interpessoal 


\section{INTRODUCCIÓN}

Según la OMS (2014) la salud mental está vinculada con el bienestar psicológico; siendo esta es un estado de bienestar con el que el individuo enfrenta el estrés de la vida comunitaria y familiar, como consecuencia de las capacidades del individuo; siendo parte importante en la salud pública. Es por esto que la salud mental o trastornos mentales, no deben ser separados de la salud en general (Posada, 2013).

Respecto al bienestar humano, este implica tener seguridad personal y en el entorno, además de acceso a bienes materiales y relaciones sociales buenas, que le permitan a la persona tener una vida digna y gozar de buena salud (Sen, 1982). La OMS (2014), resalta que tan importante es la salud mental en los individuos, además establece la necesidad existente de promover el bienestar psicológico, así como también prevenir, tratar y rehabilitar a las personas con trastornos mentales. En este sentido, países como Colombia, adoptaron lineamientos a fin de reducir las brechas existentes respecto a la salud mental, por lo que incluso promulgaron una ley; sin embargo, la inequidad respecto a salud mental, aún está presente en ese país. (Rojas, Castaño y Restrepo, 2018).

En el ámbito educativo, el docente debe de tener un óptimo bienestar psicológico; ya que su desempeño es fundamental en la política educativa. Según la Unesco (2019), hay una deficiencia de docentes bien capacitados, a nivel mundial. Respecto al bienestar psicológico docente, nivel nacional; estos tienen una gran presión, tras la exigencia que tiene Perú, por mejorar su calidad educativa. Esto hace que muchas veces se responsabilice al docente por todas las dificultades que se dan respecto a la educación. En consecuencia, muchos educadores presentan dificultades como, el poco compromiso de los padres con la actividad curricular, pérdida de autoridad con los estudiantes, desprestigio de su profesión, entre otros; generando malestar en los docentes (León y Monzón, 2019).

En el contexto local, a los docentes de la institución educativa Nº 6151 "San Luis Gonzaga", se les observa con desánimo y bajo optimismo, así como con poca colaboración en las actividades desarrolladas, evidenciando bajo control de sus emociones e impulsos; como consecuencia de esto, los padres de familia han presentado quejas por maltrato emocional hacia sus hijos. Por otro lado, se evidencia también, dejadez con respecto a sus funciones diarias, destacando la improvisación en sus clases, lo que no permite alcanzar los logros esperados. Además, tienen poca capacidad de trabajo en equipo y presentan desinterés en capacitarse y obtener nuevos conocimientos, presentando también apatía con el entorno, lo que provoca conflictos y un mal clima laboral.

Esta investigación se justifica teóricamente, ya que ahonda en las teorías respecto a las variables, además, tiene en cuenta el importante rol que cumplen los docentes en el proceso de aprendizaje y enseñanza; por lo que es importante que estos cuenten con un óptimo desempeño, a fin de alcanzar los estándares establecidos por el MINEDU. Del mismo modo, la investigación es relevante metodológicamente porque permite generar relaciones entre las variables, los instrumentos utilizados fueron validados por juicio de expertos $\mathrm{y}$ estos pueden facilitar investigaciones futuras respecto a las variables. 
El estudio tiene como objetivo general, determinar la relación que existente entre el bienestar psicológico y el desempeño en docentes de una institución educativa del Cono Sur, Lima, además se plantean cuatro objetivos específicos, los cuales determinaron las relación entre las dimensiones de la variable desempeño docente (Capacidades pedagógicas, relaciones interpersonales, responsabilidad en el desempeño y emocionalidad) con el bienestar psicológico y en docentes de una institución educativa del Cono Sur, Lima.

La pregunta de investigación general plantea: ¿Qué relación existe entre el bienestar psicológico y el desempeño en docentes de una IE del Cono Sur, Lima?, se consideraron también, problemas específicos, estos fueron: ¿Qué relación existen entre el bienestar psicológico y las dimensiones (Capacidades pedagógicas, relaciones interpersonales, responsabilidad en el desempeño y emocionalidad) en docentes de una institución educativa del Cono Sur, Lima?

La hipótesis general, planteada para esta investigación fue que: Existe una relación significativa entre el desempeño docente y el bienestar psicológico en docentes en una I.E del Cono Sur, Lima, 2019. Las hipótesis específicas plantearon si es que existe relación significativamente entre el bienestar psicológico y las dimensiones de la variable desempeño docente (Capacidades pedagógicas, emocionalidad, responsabilidad en el desempeño y relaciones interpersonales) en docentes de una I.E. en el Cono Sur, Lima, 2019.

Se han tomado como antecedentes, estudios previos nivel internacional como el de Millán et al. (2017), quienes en su investigación identificaron el efecto que tiene la dependencia universitaria en la valoración de condiciones, el estrés laboral y el bienestar psicológico de los docentes. Para esto aplicaron la Escala de Bienestar Psicológico, el inventario de percepción de estresores y un cuestionario sobre la condición de trabajo. El estudio tuvo una muestra con 903 docentes pertenecientes a 32 universidades de Venezuela. Concluyendo que la inquietud que tienen los docentes acerca del nivel académico de sus alumnos, amortigua el impacto negativo sobre el bienestar psicológico.

Se consideró también los estudios de Muñoz, Fernández y Jacott (2018) realizados en España, los autores investigaron la relación entre la satisfacción vital y el bienestar subjetivo, la muestra estuvo conformada por 180 docentes y concluyeron que la correlación es significativa y moderada, ya que se obtuvo un $r=0,547$. Por otro lado, Medina (2017), en su investigación sobre si el clima organizacional incidía en el cumplimiento y desempeño de los maestros, este fue realizado en Ecuador, obteniendo una correlación positiva baja entre ambas las variables, por lo que se concluye que se debe de utilizar y mejorar el sistema de recompensa docente, a fin de que estos perciban un mejor clima laboral.

Por su parte, Castañeda (2018) determinó el vínculo entre la evaluación de desempeño docente y el rendimiento académico en universitarios de la universidad de Zacapa, Guatemala. El estudio fue descriptivo y correlacional, la muestra comprendió 270 estudiantes y 23 docentes. Se utilizó como instrumento cuestionarios para recolectar información. Los resultados concluyeron en tuvieron índices de $92.26 \%$ y $93.07 \%$ para el desempeño docente y el rendimiento académico, respectivamente; por lo que se concluye que, entre las variables, existe una correlación significativa. 
A nivel nacional, se consideraron como antecedentes los estudios de León y Monzón (2019) quienes, en su estudio realizado en el departamento de Cajamarca, Perú determinaron la relación que tienen las dimensiones de bienestar psicológico con el síndrome de Burnout, también conocido como el desgaste profesional. Para esto, la muestra fue conformada por 42 docentes, utilizando la escala de bienestar psicológico de Ryff y el Inventario de Burnout de Maslach. Concluyendo que no existe correlación significativa entre las variables.

Rosas (2020) en su investigación realizada en el colegio Mariano Melgar; tuvo como objetivo determinar, desde la perspectiva de los alumnos, la relación entre el liderazgo del director y el desempeño docente. La muestra del estudio fue de 110 alumnos. La investigación tuvo un diseño descriptivo correlacional. Se utilizaron como instrumentos s cuestionarios relativos al liderazgo pedagógico del director y el desempeño docente. El estudio tuvo un diseño descriptivo correlacional. A partir de los resultados se concluyó que el desempeño docente y el liderazgo pedagógico del director tienen una relación significativa.

En relación al bienestar psicológico, los autores Ryff, y Singer (2008) lo defines como el esfuerzo que tiene el individuo por alcanzar su potencial individual, y es importante ya que es un indicativo de la capacidad para superar dificultades, adaptarse a nuevos entornos sociales y continuar desarrollándose. Por otro lado, Alarcón (2009) lo define como un estado de felicidad con una duración regular.

Asimismo, los autores Ryff, y Singer (2008) consideran cinco dimensiones para la variable bienestar psicológico; la primera es la auto- aceptación, considerada por los autores como el conocimiento que tiene el individuo de sí mismo, en el que este identifica sus emociones, motivaciones y quehaceres; la segunda dimensión son las relaciones positivas con otras personas, esto se define como la capacidad de interactuar y establecer relaciones significativas y duraderas; la tercera dimensión es la autonomía, para los autores, la autonomía brinda resistencia a la presión social y ayuda a mejorar la conducta; la cuarta dimensión es la capacidad de adaptación y modificación de las necesidades a entornos favorables; finalmente, la dimensión crecimiento personal, donde el individuo mejora como persona para llegar al máximo de su potencial y capacidades.

Respecto al desempeño docente, Valdés (2006) lo define como un proceso sistemático para valorar el impacto educativo de los docentes frente a sus estudiantes. Por otro lado, Fernández (2008) lo describe como el conjunto de componentes que conforman la labor docente, estas pueden ser la gestión de proyectos, reuniones con padres de familias, elaboración de material didáctico entre otros. La evaluación del desempeño docente implica una revisión organizacional en los procesos, metodologías, productos de aprendizaje, a fin de poder realizar mejoras y correcciones. Esto ofrece la oportunidad de encontrar nuevas alternativas que faciliten la enseñanza del docente (Rodríguez,1999).

Acerca de las dimensiones de desempeño docente, se han tomado en cuenta las cuatro dimensiones planteadas por Valdés (2006) siendo la primera dimensión, las capacidades pedagógicas, las cuales se refieren a las estrategias de enseñanza que tienen los docentes para poder lograr aprendizajes significativos en sus alumnos; la segunda dimensión 
es la emocionalidad, referida al nivel de satisfacción que se tiene frente a la acción que realizada por el maestro; la tercera dimensión es la responsabilidad en el desempeño, referido a la responsabilidad del docente frente a las actividades y labores académicas, como la puntualidad en las clases, entrega de documentos, entre otros; por último, la cuarta dimensión, relaciones interpersonales, refiera la capacidad de tener relaciones interpersonales, mantener una comunicación asertiva tanto con los alumnos, padres de familia y otros docentes.

\section{MÉTODO}

Se empleó el enfoque cuantitativo, fundamentado en el paradigma positivista. Por otro lado, el método utilizado fue el hipotético deductivo, la investigación es de tipo básica, ya que brinda un esquema organizado de conocimientos científicos. Además, tiene un diseño no experimental, correlacional y transversal, que tiene como finalidad determinar en qué grado dos o más variables están relacionadas; el diseño es no experimental ya que no hay manipulación de variables, por lo que estas son observadas en un contexto natural (Hernández, Fernández y Baptista 2014). La población censal estuvo conformada por 90 docentes pertenecientes a la I.E. San Luis Gonzaga, ubicada en el distrito de San Juan de Miraflores. La muestra fue censal, ya que el total de individuos es un número razonable para realizar la investigación.

Se utilizó la técnica de la encuesta y como instrumentos se aplicaron dos cuestionarios respecto a las variables de investigación, uno sobre el bienestar psicológico y otro sobre el desempeño docente, respecto a la variable desempeño docente, se basó en la escala de Bienestar Psicológico de Carol Ryff. Se utilizó el coeficiente de V de Aiken para determinar la validez de los instrumentos para el bienestar psicológico. Además, para ambos instrumentos fue aplicado el juicio de expertos. Respecto a la confiabilidad de los instrumentos para la variable bienestar psicológico y desempeño docente se presenta un alfa de Cronbach de ,84, en ambos casos.

Se realizó una prueba piloto con 30 individuos de un centro de estudios con características similares a la institución educativa en estudio. Se obtuvo un alfa de Cronbach de ,916 y ,858 para la escala de Bienestar Psicológico y desempeño docentes, respectivamente; demostrando una alta confiabilidad en ambos casos.

Se utilizó un paquete estadístico para analizar estadísticamente los datos recolectados; en relación a la estadística inferencial, fue empleado el coeficiente de correlación de Sperman, esto permitió analizar todas las posibles relaciones entre las variables, por otro lado, permitió también analizar las relaciones con las dimensiones determinadas

\section{RESULTADOS Y DISCUSIÓN}

En este apartado se muestran los resultados inferenciales acerca de las pruebas de hipótesis sobre las dimensiones acerca de la escala de Bienestar Psicológico y desempeño docentes.

\section{Prueba de hipótesis general}

En la Tabla 1, de acuerdo al resultado del coeficiente de correlación, la relación entre las variables bienestar psicológico y desempeño docente, es 575, esto indica una correlación positiva y moderada entre las variables estudiadas. Y el p-valor $=, 000$ que es menor a $\alpha=, 05$ por lo cual se rechaza la hipótesis nula, por ende, se puede afirmar la H1: Existe relación significativa entre el bienestar psicológico y el desempeño docente. 
Tabla 1. Prueba de correlación entre las variables bienestar psicológico y desempeño docente.

\begin{tabular}{lllrr}
\hline & & Bienestar psicológico & Desempeño docente \\
\hline \multirow{2}{*}{ Rho de Spearman } & Bienestar & Coeficiente de correlación & 1,000 &, $575^{* *}$ \\
& Psicológico & Sig. (bilateral) &. &, 000 \\
& & N & 90 & 90 \\
& Desempeño & Coeficiente de correlación &, $575^{* *}$ & 1,000 \\
& Docente & Sig. (bilateral) &, 000 & $\cdot$ \\
& $\mathrm{N}$ & 90 & 90 \\
\hline
\end{tabular}

**. La correlación es significativa en el nivel 0,01 (2 colas).

\section{Prueba de hipótesis específica 1}

Según la Tabla 2, los resultados de Rho de Spearman, entre las variables bienestar psicológico y la dimensión capacidades pedagógicas es ,428, por lo que se obtiene una correlación positiva y moderada. Por otro lado, la significancia de p-valor
$=, 000$ es menor a $\alpha=, 05$ por lo cual se rechaza la hipótesis nula. Por lo que se afirma la H1: Existe relación significativa entre el bienestar psicológico y dimensión capacidades pedagógicas de los docentes.

Tabla 2. Prueba de correlación entre la variable bienestar psicológico y la dimensión capacidades pedagógicas.

\begin{tabular}{lllrr}
\hline & & \multicolumn{1}{c}{$\begin{array}{c}\text { Bienestar } \\
\text { psicológico }\end{array}$} & $\begin{array}{c}\text { Capacidades } \\
\text { pedagógicas }\end{array}$ \\
\hline \multirow{2}{*}{ Rho de Spearman } & $\begin{array}{l}\text { Bienestar } \\
\text { psicológico } \\
\text { Coeficiente de }\end{array}$ & Coeficiente de correlación & 1,000 &, $428^{* *}$ \\
& Sig. (bilateral) & $\mathrm{N}$ &. &, 000 \\
Capacidades & Coeficiente de correlación &, 40 & 1,000 \\
& pedagógicas & Sig. (bilateral) &, 000 & 90 \\
& $\mathrm{~N}$ & 90 & 90 \\
\hline
\end{tabular}

**. La correlación es significativa en el nivel 0,01 (2 colas).

\section{Prueba de hipótesis específica 2}

En la Tabla 3, los resultados del coeficiente de correlación Rho de Spearman, entre las variables bienestar psicológico y dimensión emocionalidad es ,465 por lo que la correlación es positiva y moderada entre la variable y la dimensión. Y la significancia de $\mathrm{p}$ - valor $=, 000$ que es menor a $\alpha=, 05$ por lo cual se rechaza la hipótesis nula, por lo que se puede afirmar la H2: Existe relación significativa entre el bienestar psicológico y dimensión emocionalidad de los docentes. 
Tabla 3. Prueba de correlación entre la variable bienestar psicológico y la dimensión emocionalidad.

\begin{tabular}{lllrr}
\hline & & Bienestar psicológico & Emocionalidad \\
\hline \multirow{2}{*}{ Rho de Spearman } & Bienestar & Coeficiente de correlación & 1,000 &, $465^{* *}$ \\
& Psicológico & Sig. (bilateral) &. &, 000 \\
& & $\mathrm{~N}$ & 90 & 90 \\
& \multirow{2}{*}{ Emocionalidad } & Coeficiente de correlación &, $465^{* *}$ & 1,000 \\
& & Sig. (bilateral) &, 000 & $\cdot$ \\
& $\mathrm{N}$ & 90 & 90 \\
\hline
\end{tabular}

**. La correlación es significativa en el nivel 0,01 (2 colas).

\section{Prueba de hipótesis específica 3}

Según la Tabla 4, los resultados del coeficiente de correlación, entre las variables bienestar psicológico y la dimensión responsabilidad en el desempeño, es ,541 por lo que se puede afirmar que la correlación es positiva y moderada entre la variable y la dimensión. Por otro lado, la significancia de p-valor $=, 000$ que es menor a $\alpha=, 05$ por lo cual se rechaza la hipótesis nula. Es por esto que se afirma la H3: Existe relación significativa entre el bienestar psicológico y dimensión responsabilidad en el desempeño.

Tabla 4. Prueba de correlación entre la variable bienestar psicológico y la dimensión emocionalidad.

\begin{tabular}{lllrr}
\hline & & Bienestar psicológico & Responsabilidad \\
\hline \multirow{2}{*}{ Rho de Spearman } & Bienestar & Coeficiente de correlación & 1,000 &, $541^{\star *}$ \\
& Psicológico & Sig. (bilateral) & .000 & 90 \\
& & $\mathrm{~N}$ &, $541^{\star *}$ &, 000 \\
& \multirow{2}{*}{ Responsabilidad } & Coeficiente de correlación & 90 & 1,000 \\
& & Sig. (bilateral) & N & 90 \\
\hline
\end{tabular}

**. La correlación es significativa en el nivel 0,01 (2 colas).

\section{Prueba de hipótesis específica 4}

Según la Tabla 5, el resultado del coeficiente de correlación Rho de Spearman es ,528 entre las variables bienestar psicológico y dimensión relaciones interpersonales, por lo que se puede afirmar que existe una correlación positiva $y$ moderada entre la variable y la dimensión. Por otro lado, la significancia de $\mathrm{p}$-valor $=, 000$ que es menor a $\alpha=, 05$ por lo cual se rechaza la hipótesis nula, afirmando la H4: Existe relación significativa entre el bienestar psicológico y dimensión relaciones interpersonales de los docentes. 
Tabla 5. Prueba de correlación entre la variable bienestar psicológico y la dimensión emocionalidad.

\begin{tabular}{|c|c|c|c|c|}
\hline & & & Bienestar psicológico & $\begin{array}{c}\text { Relaciones } \\
\text { Interpersonales }\end{array}$ \\
\hline \multirow[t]{6}{*}{ Rho de Spearman } & \multirow{3}{*}{$\begin{array}{l}\text { Bienestar } \\
\text { Psicológico }\end{array}$} & Coeficiente de correlación & 1,000 &, $528^{\star \star}$ \\
\hline & & Sig. (bilateral) & . &, 000 \\
\hline & & $\mathrm{N}$ & 90 & 90 \\
\hline & \multirow{3}{*}{$\begin{array}{l}\text { Relaciones } \\
\text { Interpersonales }\end{array}$} & Coeficiente de correlación &, $528^{* *}$ & 1,000 \\
\hline & & Sig. (bilateral) & ,000 & . \\
\hline & & $\mathrm{N}$ & 90 & 90 \\
\hline
\end{tabular}

**. La correlación es significativa en el nivel 0,01 (2 colas).

\section{Análisis}

Que existe una relación positiva y moderada entre las variables bienestar psicológico y desempeño docente en una institución educativa del cono Sur de Lima.

Se concluye que existe una relación positiva y moderada, entre la variable bienestar psicológico y la dimensión capacidades pedagógicas en una institución educativa del cono Sur de Lima.

Se concluye que existe relación positiva $y$ moderada, entre la variable bienestar psicológico y la dimensión emocionalidad en una institución educativa del cono Sur de Lima.

Se concluye que existe una relación positiva moderada entre la variable bienestar psicológico y la dimensión responsabilidad en el desempeño en una institución educativa del cono Sur de Lima.

Se concluye que existe una relación positiva y moderada entre el bienestar psicológico y la dimensión relaciones interpersonales en una institución educativa del cono Sur de Lima.

\section{Discusión}

Los resultados de León y Monzón (2019) permitieron inferir las áreas temáticas que deben ser consideras en futuras investigaciones ya que tuvieron como conclusión que no existía una correlación entre el bienestar psicológico de los docentes y el síndrome de Burnoout, o también llamado desgaste profesional. Por otro lado, su estudio fue una guía metodológica ya que utilizaron también, la escala de bienestar psicológico de Ryff; esto permitió profundizar en su uso e instrumentos utilizados.

Por otro lado, Millán et al. (2017) quienes en su investigación identificaron el efecto que tiene la dependencia universitaria en el estrés laboral, valoración de condiciones y el bienestar psicológico de los docente, permitieron definir la metodología a utilizar, ya que aplicaron la Escala de Bienestar Psicológico, mismo que fue utilizado en la presente investigación. Por otro lado, los resultados obtenidos permitieron plantear la realidad problemática de los docentes, los cuales serían el grupo objetivo del presente estudio. Los autores en su investigación concluyeron que la inquietud que tienen los docentes acerca del nivel académico de sus alumnos, amortigua el impacto negativo sobre el bienestar psicológico, lo que refiere que los docentes ven influido su bienestar psicológico por más de 
una variable, los cuales podrían ser considerados en una próxima investigación. También, los resultados de Castañeda (2018) permitieron inferir las líneas de investigación y áreas temáticas que deben ser consideradas, ya que el autor determinó el vínculo entre la evaluación de desempeño docente y el rendimiento académico de universitarios de la universidad de Zacapa, Guatemala.

La definición del autor Valdés (2006) sobre la variable desempeño docente, fue considerada como teoría base para la presente investigación del mismo modo, las dimensiones que este autor consideró para la variable; es así que en la presente investigación se consideraron las capacidades pedagógicas, la emocionalidad, la responsabilidad en el desempeño y las relaciones interpersonales, Estas, fueron utilizadas como base para la investigación y planteamiento de objetivos específicos.

En el estudio de Rosas (2020) el autor evidenció que el liderazgo transformacional influye de manera significativa en el clima laboral y el desempeño docente, estos resultados permiten inferir que además de las variables consideradas, es necesario realizar futuras investigaciones considerando las variables presentados por el autor. Por otro lado esta investigación esta investigación en su parte metodológica utilizó como instrumento el cuestionario de desempeño docente, mismo que fue utilizado en la presente investigación, por lo que se puede reafirmar su confiabilidad y validez.

\section{CONCLUSIONES}

Según los resultados obtenidos se concluye que existe una relación positiva y moderada entre el bienestar psicológico y el desempeño docente en una institución educativa del Cono Sur de Lima. Asimismo, existe una relación positiva y moderada entre la variable bienestar psicológico y las dimensiones del desempeño docente: capacidades pedagógicas, emocionalidad, responsabilidad en el desempeño y relaciones interpersonales; es así que para mejorar el desempeño docente es importante la implementación y desarrollo de programas que favorezcan el bienes psicológico docente, permitiéndoles tener una mejor gestión de sus emociones y control del estrés; por otro lado, es importante que las instituciones educativas reduzcan las sobre carga laboral docente $\mathrm{y}$ establezcan políticas y directivas que ayuden a minimizarla.

\section{REFERENCIAS}

Alarcón, R. (2009). Psicología de la felicidad. Introducción a la psicología positiva. Lima: Universidad Ricardo Palma

Castañeda, M. (2018). Relación del desempeño docente y el rendimiento académico de los estudiantes. Revista Ciencia Multidisciplinaria CUNORI, 2(1), 41-46. doi: /10.36314/cunori. v2i1.51

Fernández, J. (2008). Desempeño docente y su relación con orientación a la meta, estrategias de aprendizaje y autoeficacia (Tesis de Posgrado). PUCP, Lima-Perú

Hernández, R. y Mendoza, C. (2018). Metodología de la investigación. Las rutas cuantitativa, cualitativa y mixta, Ciudad de México, México: Editorial Mc Graw Hill Education, Año de edición: 2018, ISBN: 978-1-4562-6096-5, 714 p

Hernández, R.; Fernández, J. y Baptista, L. (2014). Metodología de la investigación (5ta edición) México: Mc Graw Hill

Medina, D. (2017). Clima organizacional en el desempeño docente de la Unidad Educativa Particular Santo Domingo de Guzaman - Quito. (tesis de maestria). Ecuador. Recuperado de http://repositorionew.uasb.edu. ec/bitstream/10644/5611/1/T2266-MDTHMedina- Incidencia.pdf 
Millán, A., Calvanese, N., y D'Aubeterre, M. (2017). Condiciones de trabajo, estrés laboral, dependencia universitaria y bienestar psicológico en docentes universitarios. 15(1), 195-218

Monzón Portilla, R. J., y León Alva, K. J. (2019). Bienestar Psicológico y Síndrome de Burnout en Docentes de las Instituciones Educativas de Jornada Escolar Completa de Dos Distritos de la Provincia de Cajamarca-2017. REVISTA PERSPECTIVA, 20(3), 320-326. https://doi. org/10.33198/rp.v20i3.00044

Muñoz, E., Fernández A., y Jacott, L. (2018). Bienestar subjetivo y satisfacción vital del profesorado. REICE. Revista Iberoamericana sobre Calidad, Eficacia y Cambio en Educación, 2018, 16(1), 105-117. https://doi.org/10.15366/ reice2018.16.1.007. Universidad Autónoma de Madrid, España. Recuperado de https://revistas. uam.es/index.php/reice/article/view/9030/9281

Organización Mundial de la Salud. (2014). Promoción de la salud mental. Conceptos, evidencia emergente, práctica: Informe compendiado. Ginebra: Organización Mundial de la Salud; p. 14-5

Posada, J. (2013). La salud mental en Colombia. Biomédica; 33 (4): 497-498. Disponible en: [Internet] [citado el 9 de enero de 2021] http://www.scielo.org.co/scielo. php?script $=$ sci_arttext \& pid=S 0120 $41572013000400001 \& \operatorname{lng}=$ en
Rodríguez, I., (1999). El desempeño de los docentes de las Escuelas Básicas del Distrito Escolar No 4 del Estado Aragua. Universidad de Carabobo, Venezuela

Rojas, L., Castaño, G., y Restrepo, D. (2018). Salud mental en Colombia. Un análisis crítico. CES Medicina, 32(2),129-140. ISSN: 01208705. Disponible en: https://www.redalyc.org/ articulo.oa?id=261157250007

Ryff, C. y Singer, B. (2008). Know Thyself and Become What You Are: A Eudaimonic Approach to Psychological Well-Being. Journal of happiness studies, (1), 13. Recuperado en: http://search.ebscohost.com/login. aspx?direct $=$ true $\& d b=$ edsbl\&AN=RN222540 $608 \&$ lang $=$ es \&site $=$ eds-live

Rosas Hostos, E. F. (2020). Liderazgo pedagógico directivo y desempeño docente en una Institución Educativa de Lima Metropolitana. REVISTA CONCIENCIA EPG, 1(1), 53-63. https://doi.org/10.32654/concienciaepg.1-1.5

Sen, A., (1982) Choice, Welfare and Measurement. Oxford, Blackwell

Unesco (2019). What UNESCO does in teacher development. Recuperado de https://en.unesco. org/themes/teachers/action

Valdés, V., (2006) Evaluación del Desempeño docente. Ponencia presentada por Cuba. Encuentro Iberoamericano sobre Evaluación del Desempeño docentea 